\title{
Two Distinct Pathways can Control Expression of the Gene Encoding the Drosophila Antimicrobial Peptide Metchnikowin
}

\author{
Elena A. Levashina, Serge Ohresser, Bruno Lemaitre and \\ Jean-Luc Imler*
}

Réponse Immunitaire et Développement chez les Insectes, UPR 9022 du CNRS Institut de Biologie Moléculaire et Cellulaire, 15 rue René Descartes, 67000 Strasbourg France

\begin{abstract}
Metchnikowin is a recently discovered proline-rich peptide from Drosophila with antibacterial and antifungal properties. Like most other antimicrobial peptides from insects, its expression is immune-inducible. Here we present evidence that induction of metchnikowin gene expression can be mediated either by the TOLL pathway or by the imd gene product. We show that the gene remains inducible in Toll-deficient mutants, in which the antifungal response is blocked, as well as in imd mutants, which fail to mount an antibacterial response. However, in Tolldeficient;imd double mutants, metchnikowin gene expression can no longer be detected after immune challenge. Our results suggest that expression of this peptide with dual activity can be triggered by signals generated by either bacterial or fungal infection. Cloning of the metchnikowin gene revealed the presence in the $5^{\prime}$ flanking region of several putative cis-regulatory motifs characterized in the promoters of insect immune genes: namely, Rel sites, GATA motifs, interferon consensus response elements and NF-IL6 response elements. Establishment of transgenic fly lines in which the GFP reporter gene was placed under the control of $1.5 \mathrm{~kb}$ of metchnikowin gene upstream sequences indicates that this fragment is able to confer full immune inducibility and tissue specificity of expression on the transgene.
\end{abstract}

(C) 1998 Academic Press Limited

Keywords: Drosophila immunity; antimicrobial peptides; metchnikowin; Rel; TOLL pathway

\section{Introduction}

Insects have long been known to be particularly resistant to microbial infections. This resistance is due to an efficient host defense system that comprises the proteolytic cascades leading to coagulation and melanization of the hemolymph, the phagocytosis and encapsulation of invading microorganisms by blood cells, and the secretion into the blood of a battery of potent antimicrobial peptides (for a recent review see Hoffmann \& Reichhart, 1997). Infection leads to the rapid and transient synthesis of these peptides, mainly by the fat body, a functional equivalent of the mammalian liver. Recent studies indicate that in Drosophila at least

Abbreviations used: ORF, open reading frame; GFP, green fluorescent protein from Aequonea victoria; DIF, DORSAL-related immunity factor; IRF-1, interferon regulatory factor 1 . seven distinct peptides, and their isoforms, participate in the humoral immune response. Five of these peptides, cecropins (Kylsten et al., 1990), diptericin (Wicker et al., 1990), drosocin (Bulet et al., 1993), attacin (Asling et al., 1995) and insect defensin (Dimarcq et al., 1994), are selectively active against bacteria, whereas drosomycin, is solely active against fungi (Fehlbaum et al., 1994). The seventh and most recently discovered molecule, metchnikowin, is unique among the antimicrobial peptides of Drosophila in that it is active against both bacteria and fungi (Levashina et al., 1995).

The study of the regulatory mechanisms controlling the rapid synthesis of the antimicrobial peptides after immunological challenge has become a field of intense research. Analysis of the humoral immune response in different mutant strains of Drosophila has led to the definition of two distinct pathways regulating antimicrobial peptide gene expression. Lemaitre et al. (1995) have recently 
described a recessive mutation, immune deficiency (imd), which impairs the inducibility of the genes encoding antibacterial peptides, while only marginally affecting the inducibility of drosomycin. This gene has not yet been cloned. On the other hand, these authors have shown that the well characterized TOLL pathway (Morisato \& Anderson, 1995) controls drosomycin gene expression. During embryonic dorsoventral patterning in Drosophila, the TOLL receptor is activated by a processed form of the product of the gene spaetzle (spz). A cascade of proteolytic cleavages involving the products of the genes gastrulation defective ( $(g d)$, snake $(s n)$ and easter (ea), which all code for secreted serine proteases, results in the release of active SPAETZLE in the perivitelline fluid on the ventral side of the embryo (Morisato \& Anderson, 1995). Activation of TOLL results in the release of the Rel protein DORSAL from the inhibitor CACTUS. The cytoplasmic proteins TUBE and PELLE intercalate between TOLL and CACTUS in this pathway. Interestingly, this signaling cascade bears striking structural and functional similarity to the activation cascade of the Rel protein NF- $\kappa B$ in mammals: CACTUS is a structural homologue

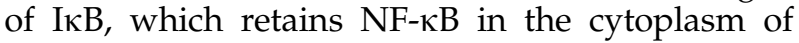
mammalian cells (Siebenlist et al., 1994). The intracytoplasmic domain of TOLL shows sequence homology with the corresponding domain in the type I interleukin-1 (IL-1) receptor (Gay \& Keith, 1991); and the kinase PELLE is related to the kinase IRAK, which interacts with the IL-1 receptor (Cao et al., 1996). In mutants deficient for spz, Toll $(T l)$, tube $(t u b)$ or pelle $(p l l)$, drosomycin inducibility is severely reduced, while the induction of diptericin and drosocin is not affected. Furthermore, in $T l$ gain-of-function mutants $\left(T l^{D}\right)$, as well as in mutants deficient for the inhibitor CACTUS, drosomycin is expressed in the absence of immune challenge (Lemaitre et al., 1996). The TOLL pathway therefore appears necessary and sufficient for the control of drosomycin expression. It also appears necessary for inducibility of cecropin, defensin and attacin, since expression of these genes is reduced after immune challenge in loss-of-function mutants of $s p z, T l, p l l$ or $t u b$. However, none of these three peptides is expressed in the absence of immune stimulation in $T l^{D}$ or cactus (cact)-deficient mutants, indicating that the TOLL pathway is not sufficient for the control of their expression (Lemaitre et al., 1996).

The importance of the TOLL and IMD pathways in the Drosophila host defense system has been illustrated by experiments in which mutant flies were challenged with fungi or bacteria. $\mathrm{Tl}$-deficient flies rapidly succumb to fungal, but not bacterial, infection, whereas imd mutants resist fungi but are markedly susceptible to bacteria (Lemaitre et al., 1995, 1996). These results are consistent with our present knowledge of the functions of the antimicrobial peptides in Drosophila, since $\mathrm{Tl}$ controls expression of the antifungal peptide gene drosomycin, while imd appears to control, or to partici- pate in the control of, the antibacterial peptides. Here, we have investigated the regulation of expression of the newly discovered peptide metchnikowin, which has both antibacterial and antifungal activity (Levashina et al., 1995). We report that the regulation of this peptide is unique compared to the other peptides, in that it appears to be regulated independently by the TOLL and the IMD pathways. We also report cloning of the metchnikowin gene and sequencing of $1.5 \mathrm{~kb}$ of upstream non-transcribed sequences. We show by functional studies using transgenic fly lines that this fragment contains the metchnikowin promoter.

\section{Results}

\section{Transcription of the metchnikowin gene}

We have previously shown that metchnikowin expression is induced upon immune challenge in Drosophila adults, and reaches a maximum level 14 hours after septic injury (Levashina et al., 1995). As stated in the Introduction, two pathways have been shown to participate in the regulation of the humoral immune response in Drosophila. The first pathway is defined to date only by the imd gene product, which controls antibacterial peptide gene expression (Lemaitre et al., 1995), whereas the TOLL pathway regulates the expression of the antifungal peptide drosomycin (Lemaitre et al., 1996). In order to determine if these pathways regulate metchnikowin expression, we performed Northern blots with RNA prepared from wild-type or mutant flies. Two types of mutants affecting the TOLL pathway were analyzed: first, strains carrying strong loss-of-function mutations of $g d$, snk, ea, $s p z, T l, t u b, p l l$ and dorsal ( $d l)$, which are known to block the dorsoventral signaling pathway resulting in dorsalized embryos; and second, gain-of-function mutations in $\mathrm{Tl}\left(\mathrm{Tl}^{\mathrm{D}}\right)$ and loss-of-function mutations in cact that are strongly ventralizing in embryos. Mutants of the first type, as well as the imd mutant flies in which induction of the genes encoding antibacterial peptides is impaired, were challenged by an injection of bacteria before RNA extraction six hours later, whereas ventralizing mutants were analyzed in the absence of challenge.

As shown in Figure 1, metchnikowin remained significantly inducible by septic injury in Drosophila adults carrying a loss-of-function allele of the $\mathrm{Tl}$ gene. The variability from experiment to experiment, which can be observed in all mutant backgrounds, was also observed for all other peptides, and most probably reflects the complexity of this in vivo system (Lemaitre et al., 1996). Nonetheless, only in one out of seven experiments performed was metchnikowin inducibility reduced to $50 \%$ of the wild-type inducibility (Figure 1, compare lanes 2 and 9). In all other experiments, the inducibility of metchnikowin gene expression in $\mathrm{Tl}$-deficient mutants was similar or superior to the level of inducibility in wild-type flies. These experiments were extended to other mutations affecting the 


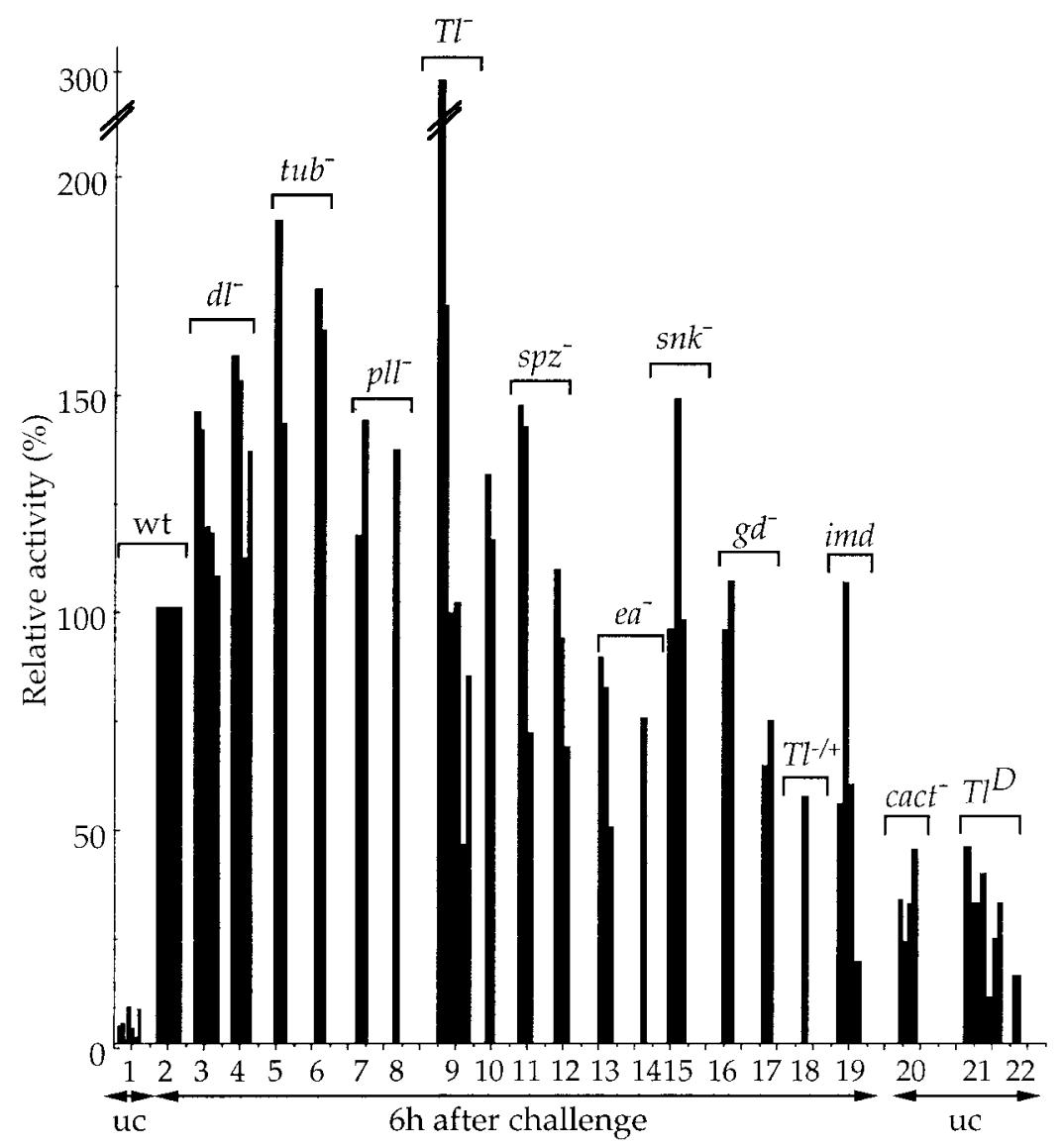

Figure 1. Quantification of metchnikowin expression in wildtype and mutant adults. Total RNA prepared from control (unchallenged: uc) or bacteria-challenged (six hours) wild-type (wt) or mutant adult flies were analyzed by Northern blot using a metchnikowin cDNA probe. The signals on several Northern blots were quantified with a Bio-Imager system. In each experiment the blot was also probed with the ribosomal protein rp49 cDNA and the signals for metchnikowin were normalized with the corresponding value of the rp49 signal. The levels of expression in wild-type adults six hours after bacterial challenge were taken arbitrarily as 100 and the results are presented as relative activities. Each bar corresponds to an independent experiment. Lanes are as follows: 1 and $2, \mathrm{Or}^{\mathrm{R}} ; 3, d^{1} /$ $d l^{1} ; 4, d l^{T} / D f(d l) ; 5, t u b^{238} / t_{u} b^{118} ; 6$, tub $b^{3} /$ tub $^{118} ; 7, \mathrm{pll}^{078} / \mathrm{pll}^{21} ; 8, \mathrm{pll}^{178} /$ $\mathrm{pll}^{r m 8} ; 9, \mathrm{Tr}^{r 632} / \mathrm{Tl}^{1-R X A}\left(29^{\circ} \mathrm{C}\right) ; 10$ $\mathrm{Tl}^{r 444} / \mathrm{Tl}^{9 \mathrm{QRE}} ; 11, \mathrm{spz}^{197} / \mathrm{spz}^{197} ; 12$, $s p z^{r m 7} / \mathrm{spz}^{r m 7} ; 13, \mathrm{ea}^{1} / \mathrm{ea}{ }^{1} ; 14, \mathrm{ea}^{818} /$ ea $a^{818} ; 15, \mathrm{snk}^{073} / \mathrm{snk}^{073} ; 16, \mathrm{gd}^{7} / \mathrm{g} d^{7}$; 17, $g d^{8} / g d^{8} ; 18, T l^{1-R X A} /+; 19$, imd/imd; 20, cact $^{A 2} /$ cact $^{A 2} ; \quad 21$, $\mathrm{Tl}^{10 b} /+$.
TOLL pathway. Mutants deficient in the $g d$, snk and ea genes, which code for serine proteases acting upstream in the pathway during embryonic development, and which are not essential for the control of drosomycin expression (Lemaitre et al., 1996), showed a wild-type response to bacterial challenge (Figure 1, compare lanes 13 to 17 to lane 2 ). In addition, loss-of-function mutations in $t u b, p l l$ or spz, which affect drosomycin expression (Lemaitre et al., 1996), clearly did not result in decreased inducibility of metchnikowin (Figure 1, compare lanes 5 to 12 to lane 2). On the contrary, metchnikowin RNA expression level in these induced mutants flies was often higher than in wild-type. Similar results were obtained with $d l$ deficient mutant flies (Figure 1, compare lanes 3 and 4 to lane 2). Although we do not have any explanation to date for these variations, we note that similar upregulation of diptericin mRNA in these mutants has been observed (Lemaitre et al., 1996). Altogether, these data indicate that metchnikowin gene expression can be turned on by bacterial challenge in the absence of a functional TOLL pathway.

We next examined metchnikowin gene expression in imd mutants, in which the antibacterial response is affected. We observed that metchnikowin gene expression was slightly reduced in imd mutants. In one out of four experiments, the inducibility of metchnikowin in imd mutants was reduced by fivefold, in another experiment it was equivalent to wild-type inducibility, whereas in the remaining two experiments, induction level was roughly half of wild-type (Figure 1, compare lanes 19 and 2). Therefore, we conclude that metchnikowin expression remains significantly inducible by bacterial challenge in the absence of a functional imd gene product.

These data suggest either that in immune-challenged flies metchnikowin expression is not regulated by the IMD or TOLL pathway, or that both pathways can control metchnikowin gene expression. To discriminate between these two possibilities, we examined the inducibility of metchnikowin gene expression six hours after septic injury in double mutants for Toll and imd, and observed that in this context the induction of metchnikowin is completely abolished (Figure 2). These results sustain the hypothesis that both pathways participate in the regulation of metchnikowin expression.

In order to study further the role of the TOLL pathway in metchnikowin gene regulation, we then analyzed metchnikowin gene expression in the absence of immune challenge in $T l^{D}$ gain-offunction and cact-deficient adults. Previous studies had shown that drosomycin is constitutively expressed in these mutants (Lemaitre et al., 1996), thus suggesting that the TOLL pathway is sufficient for regulation of drosomycin expression. Strikingly, a significant expression of metchniko- 


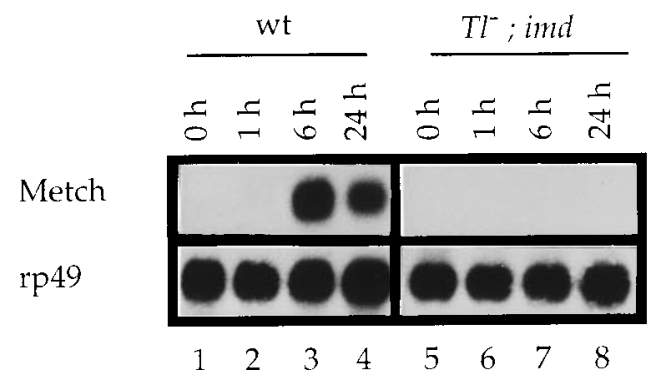

Figure 2. Transcriptional profile of metchnikowin in wild-type or imd;Tl-deficient double mutant Drosophila adults. A representative Northern blot of total RNA extracted from control (0 hour; lanes 1 and 5) or bacteria-challenged $(1,6$ or 24 hours after challenge) wildtype (wt) or mutant adult Drosophila is shown. The blot was first probed with the metchnikowin cDNA, and after dehybridization, with the ribosomal protein rp49 cDNA. Lanes 1 to 4, wild-type Drosophila (OregonR strain); lanes 5 to 8 , imd;Tl-deficient double mutant.

win RNA was observed in the absence of immune challenge in cact-deficient mutants (Figure 1, compare lanes 20 to lane 1) and $T l^{D}$ mutants (Figure 1, compare lanes 21 to 22 to lane 1 ). In all four experiments performed with cactdeficient flies, the metchnikowin expression level was between $25 \%$ and $40 \%$ of that in immunechallenged wild-type flies. Similar results were obtained in $T l^{D}$ mutant flies, with the exception of two out of seven experiments in which metchnikowin RNA expression level only reached 15\% of that in immune-challenged wild-type flies. We conclude from these data that activation of the TOLL pathway is sufficient to induce a significant level of metchnikowin expression.

These results indicate that metchnikowin regulation differs from that of the other antimicrobial peptides of Drosophila characterized to date, in that it remains inducible in Toll-deficient and in imd mutant flies. We have therefore compared expression of metchnikowin in different mutant backgrounds with that of the antibacterial peptides diptericin and cecropin A1, and with that of the antifungal peptide drosomycin. For that purpose, Northern blots representing the populations of mRNAs from wild-type, $\mathrm{Tl}$-deficient, imd, cactdeficient and $T l^{D}$ mutant flies were repeatedly dehybridized and successively probed with metchnikowin, drosomycin, diptericin and cecropin A1 probes. Figure 3 shows that regulation of metchnikowin gene expression in immune challenged $\mathrm{Tl}$ deficient mutants is similar to that of diptericin, whereas in imd mutants it is similar to that of drosomycin. On the contrary, the regulation of cecropin A1 gene expression upon immune challenge is similar to that of drosomycin in $\mathrm{Tl}$-deficient mutants, and to that of diptericin in imd mutants. In addition, metchnikowin is expressed at a higher level than diptericin and cecropin $\mathrm{A}$ in unchal- lenged cact-deficient and $T l^{D}$ mutants, albeit at a lower level than drosomycin (Figure 3).

To summarize these results, we propose that the metchnikowin gene is unique in that it can be regulated by the IMD pathway in the absence of a functional TOLL pathway, and by the TOLL pathway in imd mutants. Thus, the particularity of metchnikowin, which has both antibacterial and antifungal activity, extends to regulation of its expression. The rest of the paper describes the isolation and organization of the promoter of the gene encoding this peptide.

\section{Cloning of the metchnikowin gene}

A genomic library of Drosophila melanogaster was screened using metchnikowin cDNA as a probe. Ten hybridization-positive clones were isolated. A $9 \mathrm{~kb}$ SalI fragment, which hybridized to the metchnikowin cDNA, was subcloned into the vector pTZ18R for restriction mapping. Subsequently, a $2.5 \mathrm{~kb}$ SalI-XhoI fragment was subcloned in M13 for sequencing (Figure 4(a)). This region consists of $1.5 \mathrm{~kb}$ of $5^{\prime}$ flanking sequences, followed by a 52codon open reading frame (ORF) corresponding to the metchnikowin precursor peptide. This ORF comprises a putative signal peptide and the 26 amino acid residue long mature metchnikowin (Figure 4(b)). No additional sequences were found in the transcribed region of the metchnikowin genomic clone in comparison to the cDNA clone (Levashina et al., 1995), indicating that the gene encoding metchnikowin is intron-less. The location of the cap site was determined by primer extension (data not shown). It is located 29 nucleotides upstream of the translation initiation codon (Figure $4(\mathrm{~b})$ ). The TATA box (TATAAAAG) is located between positions -31 and -24 with regard to the transcription start site $(+1)$, and putative polyadenylation consensus sequences are found 45 and 58 nucleotides downstream of the translation stop codon (Figure 4(b)).

As stated in the Introduction, several studies aimed at functional characterization of the promoters of the genes coding for Drosophila antimicrobial peptides have highlighted the importance of nucleotide sequence motifs related to NF- $\kappa$ B DNA binding sites (referred to as Rel sites hereafter) (reviewed by Hoffmann \& Reichhart, 1997). Computer analysis revealed three such nucleotide sequence motifs in metchnikowin gene upstream sequences. They are located on either strand of DNA, at positions -638 (upper strand), -176 (lower strand), and -63 (lower strand) (Figure 4(b)). The most distal Rel site (at position -638) resembles two Rel sites found in the promoter of the drosomycin gene (5'-GGGTTTTTCA-3' and 5'-GGGTTTTTAC-3'; L. Michaut et al., unpublished results). Interestingly, these two drosomycin Rel sites are located more than 500 base-pairs upstream from the start site, in contrast to the Rel sites in the genes encoding antibacterial peptides, which are usually found close to the coding region, 


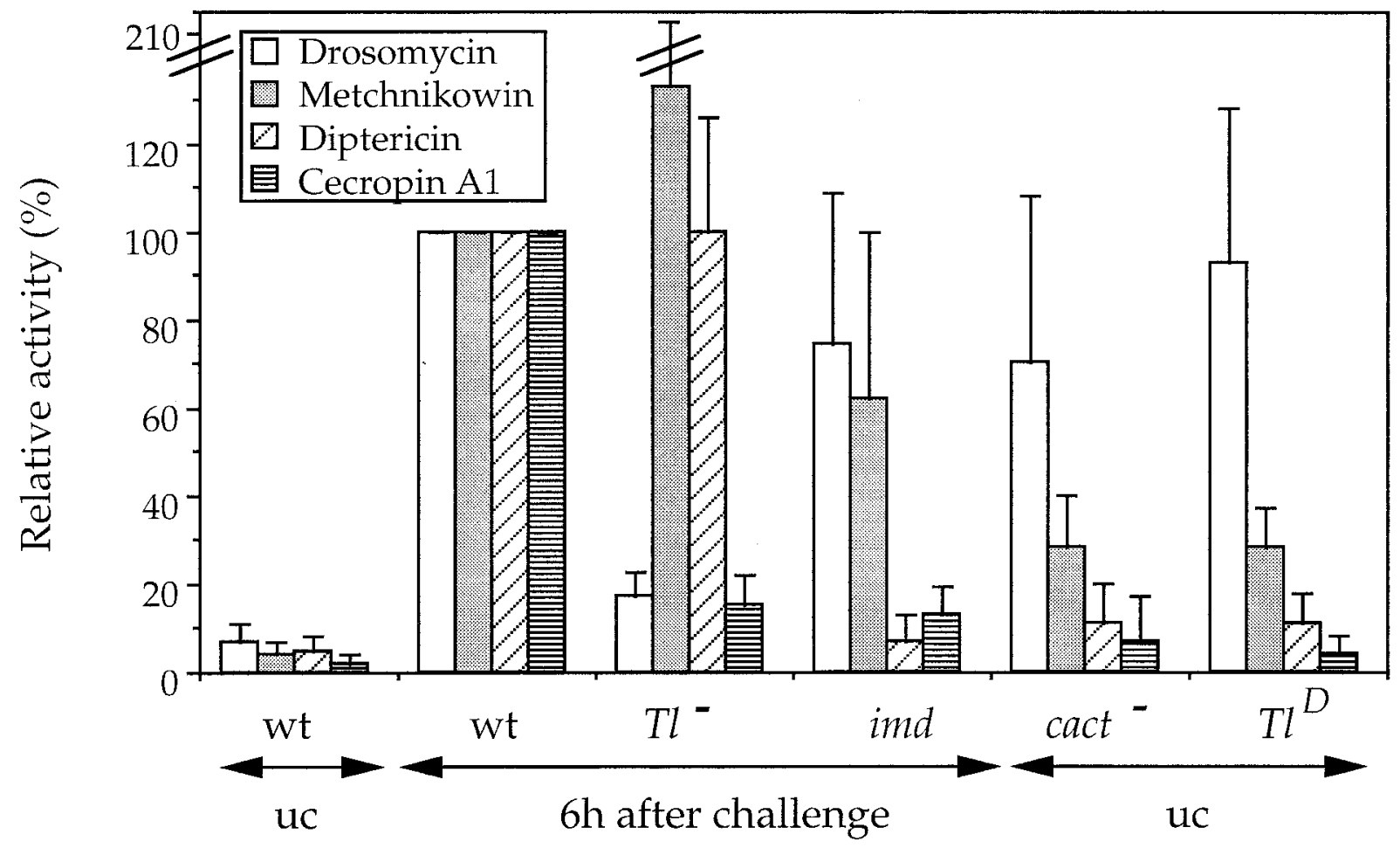

Figure 3. Comparison of drosomycin, metchnikowin, diptericin and cecropin A1 expression patterns in wild-type and mutant Drosophila adults. Northern blot with total RNA extracted from unchallenged (uc) or bacteria-challenged (six hours after challenge) wild-type (wt) or mutant flies was successively hybridized with probes corresponding to the following cDNAs: drosomycin, metchnikowin, diptericin, cecropin A1 and rp49. The signals were quantified with a Bio-Imager system. In each experiment the values for the immune peptides were normalized with the corresponding value of the rp49 signal. The level of expression of each antimicrobial peptide gene in wild-type adults after bacterial challenge was taken arbitrarily as 100 and the results are presented as relative activities. The data represent the means \pm standard deviation of four to nine independent Northern blotting experiments. Other indications: wt, Oregon R; $\mathrm{Tl}^{-}, \mathrm{Tl}^{632} / \mathrm{Tl}^{1-R X A}$; imd, imd/imd; $\mathrm{cact}^{-}$, $\mathrm{cact}^{A 2} / \mathrm{cact}^{A 2} ; \mathrm{Tl}^{\mathrm{D}}, \mathrm{Tl}^{10 B} /+$.

between positions -40 and -200 (Charlet et al., 1996; Dimarcq et al., 1994; Engstrom et al., 1993). Our analysis also revealed other consensus nucleotide sequence motifs in the metchnikowin promoter, corresponding to mammalian NF-IL6 response elements (Hocke et al., 1992), GATA sites (Orkin, 1992), and interferon consensus response elements (Georgel et al., 1995) (Figure 4(c)).

In summary, sequencing of $1.5 \mathrm{~kb}$ of DNA located upstream of the start site allowed the identification of three Rel sites. Additional studies are needed to establish the functionality of these sequence motifs and their role in the control of metchnikowin gene expression. In order to show in vivo that this $1.5 \mathrm{~kb}$ DNA fragment contains the metchnikowin promoter, we have fused it to a reporter gene, and have analyzed its function in transgenic Drosophila.

\section{Analysis of expression of a metchnikowin-GFP fusion gene}

In our first attempt to functionally characterize the metchnikowin promoter, we have constructed a reporter gene containing $1.5 \mathrm{~kb}$ of metchnikowin upstream sequences fused to the jellyfish Aequorea victoria green fluorescent protein (GFP) cDNA;
$790 \mathrm{bp}$ of $3^{\prime}$ flanking region of the drosomycin gene were used to signal transcription termination and polyadenylation. The advantage of this reporter gene is that it allows direct imaging of the fluorescent gene product in living cells without the need for prolonged and lethal histochemical staining procedures. Unlike the product of the metchnikowin gene, which is targeted by a signal sequence for secretion in the hemolymph, the GFP expressed from these constructs remains within the cells where it is synthesized. The transposon containing this reporter construct was injected into embryos, and 19 independent insertions were obtained and characterized. GFP expression was monitored qualitatively using a fluorescence stereomicroscope, or quantitatively using a fluorimeter.

Third instar larvae were pricked with a bacteriasoaked needle, and examined 12 hours later under the fluorescence microscope. A strong fluorescence could be seen in the fat body of larvae 16 hours after immune challenge (compare (a) and (b) in Figure 5). Similar results were obtained with all 19 independent lines. When adults were challenged by septic injury, a strong fluorescence developed in the fat body 24 hours later (data not shown). These data are consistent with in situ hybridization experiments, which detected abundant metchnikowin 
(a)

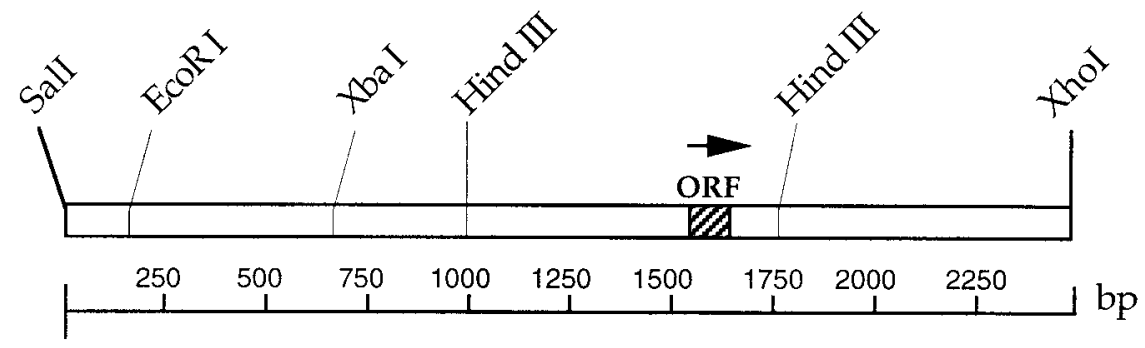

(b) TCGACTTTTCTGCCATTAGCCATCGGCTGTGATTGATTGACTAATACCGCAATTTGCCTGTCTCCCACCGATGTTTACAT 'ICACAATCTATTCCTCTGTTTACGCGCAAGCCAAACATTTATTTTTTTAATGCGCTAACCATGGAAATTGATTAATTTTAT TTGGAATTCCGTTTGATTTATTTGATTTATAGCTGCCGAAGAGAACATAAAAGGAATTTTCGTAAACTGATATTGAAGGC IGAGGTCAATTCAAATCGATTTGATAAACAATAACATTTTTATGTTTTATGTACACCTATTGTTTGTCTTGCTGGGGTTT IGGGTACTTTGCTGAAGCGGAAACTGCGTCAATCAATGCAGCTTACCCCGTTITTCCTAAACTACCAGCGTAAGTGAAGC GTTTTCAACGCCTAATGAGCCGTGACTATAACCGCTTITTTTCCTCCTAAAAAGTAAGCACACAAACAATAAAACGCAAG TGCAAAATTACTCAAAATGTCAAAATGTCGAGTCATTTAAAGGGCAGCGCCACGTTCAACCTCTTTTGCATGCCCCTTTC TGCTGCGAGAAAACTAACAAAGTGCTCTAATCGAGCCAAGGGGCAATTTCTTGTGTTGCTGCAGCTGCACTTTGCACCTC CGCATCCGTGCACCCAAAAACCCGCTTTCTAGATGTTCTCATCATGCACTGAAAAAGAATCCAAATTTTTACAAGAAATA GTTTAAAATTAGGTAATGTGAAAGATATCGGCACACGGACAGGCCGAATTATCTGTTGTAAAACTAGCTGCTCAGTTATT AAAAACATTTGTAGTTGCTGACGTTTCCATACAGAGACTAATTTTATW'TTCACGGACAGGGGTTTTCCGCTTTAATTGCT TCATTTTTGTTGCTTTATTGCGTGTATATTGCCCCACAAAACAGATATAAATCATTCGCGCATATCGTAAATGTTGGTAG AAAATGACAAACAAGAGAAAAAAGATGATTAAAAGCTTCAAGACAATCCTCTATRGGATCTGATTAAATATGAATATTTT ATTTATTAGTTTTCTTTCTGTGTACGGCTTAGAAGGCAGAAGCTGCGAGGGGCGTAGGGCAGTGGGCGTGGCTCCGTGTT GACGCATGTTGACTATGCCTRTGAATGGCTGCCGTGGT'TGTCGGTGGGTAA'TTGCAATGCAGAAAAACCAACAGGGCGC TAAAAAGGAGAGTGTTTTCGTGGGAGGTGGAGATGGTCACTGGGGGAACATAAATATTCAGCGAGAAACGTCATATTTA CATTTAGTCTAGGCTGATAATCCGGGACCGTGGGAAGTCCCCTTTGGGTGGTGCTGGCTGGGTTCCCCTGGCCACAATCG GTTATCTGCCCCCGGCTGACACTTGCCCGTCATTCATTCGGCTGCTTATCGCAGAAGCTCAAATAAAAAGTCCCCAATCT GCGACTCGTTTGTCTGGAACTGAGTATAAAAG CTCGCCATCTCAACGCTCTAAGCATCAATCAATTCCCGCCACCGAG CDNA

CCACATCGTCACCAGGGACCCATTTTCGATACGAGGCCGTCGCCCTTCAATCCTAACCAACCAAGACCGGGTCCAATTITA $\begin{array}{lllllllllllllllllllllllllllllll}P & H & R & H & Q & G & P & I & F & D & T & R & P & S & P & F & N & P & N & Q & P & R & P & G & P & I & Y\end{array}$

TTAAATTGACAGTGGGAAATCACACTGCTGATGTCGTTAACACATTGATAAAATTAATACAAT"TAAACCAAAACTGAAC stop

ATCAAAGCTTGTCATTGGGTAATTAAATTAATTCCACGCTGAAGGGGGCCAAAAGACACAGGTAACTGGAGGGCGGGCAA. $\longrightarrow$ CDNA

TTAGCAATCACAGTTTCACTGAACCAGAATTATGAGGTAGCTGCAATTGAAATTGTACCTCGCTGATTGTTCGGTGTTTA AGGCTTCTTCAATGGTATTCAATCCATAAATCATAATGGCAACTGTGATTGAGCCAACATTTGGAATGCAAAAGCACTCG AATGCAATTTAATTTGCATTTTGCATCTGCTTGGCTCGCGGGGCGATGAGTAATCACGGTTCCATCGATTGCCATTCP CTGTAGTATACTACACTTGTGCATGTATATTTAGATGCGAATAAAAATTGAGTACGCGCAAACATTTCAATGAGCTTCAC ACACAAAAACGACAATTTCTTCCTCACCACTCTCCGCTGTTTCTGTCTATTTTCAGCTGCTATCCAAGAAAAGCGCTAAG AAAAACACTTGCAAAAATTAGCAGCAGCAGCTGATGGACGAATGGCGGTTGGCGGCGTTCGGTGCGGGAGGCCGTTTCGT CTATAAATAGTC'T'TGAACACTTTCTGCGCGTGACAGCTGTGACAGCGGCCATGACGTCATTGGC'TGGATGAGCCACGTC ACGGATGCGGTATAAACTGGTCTGGCTAAGGCCAATGAAAGCATATCGCGCATACGCCCCGTTGACCCACGGCAAACAAA AACGC

(c)

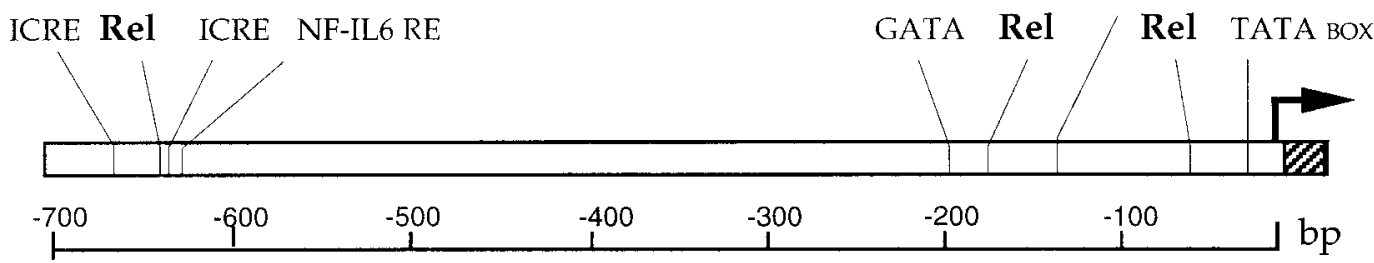

Figure 4 (legend opposite) 

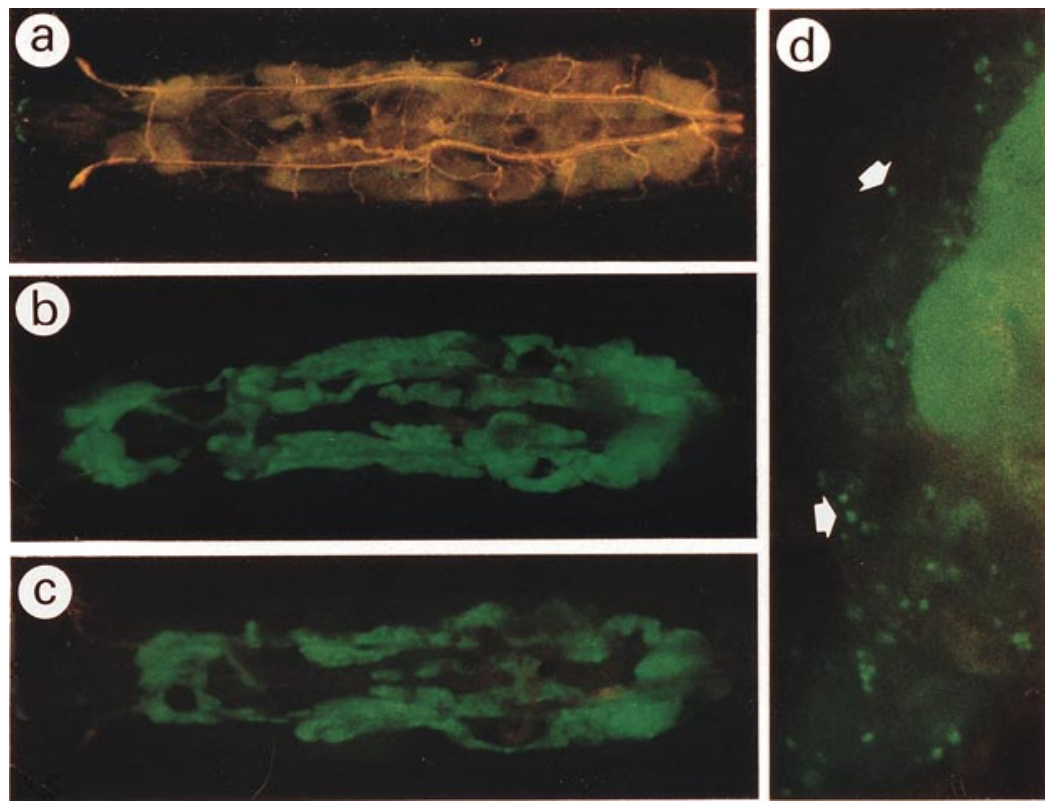

Figure 5. Inducible GFP expression in transgenic larvae containing the GFP cDNA under the control of $1.5 \mathrm{~kb}$ of metchnikowin gene upstream sequences. (a) Non-immunized transgenic larva. (b) Transgenic larva 16 hours after immune challenge with bacteria. (c) Constitutive fluorescence in the fat body of an unchallenged transgenic larva carrying one copy of the $T l^{10 b}$ gain-of-function Toll allele. (d) Fluorescent circulating hemocytes (arrows) in a transgenic larva 16 hours after bacterial challenge. Larvae were illuminated with a wavelength of $495 \mathrm{~nm}$, and visualized using a fluorescence stereomicroscope.

transcripts in the fat body of immune challenged adults (Levashina et al., 1995). In addition, when third instar larvae where pricked with a bacteriasoaked needle, fluorescent hemocytes could be seen through the cuticle 12 hours later (Figure 5(d)), but only in some larvae $(\sim 10 \%)$. This discrepancy between larvae most probably reflects the intensity of the immune response, with only the strongest responding larvae reaching the GFP protein concentration threshold required to detect fluorescence before puparation. Inducible expression of the genes encoding antimicrobial peptides in larval hemocytes has been documented for diptericin (Meister et al., 1994) and cecropin (Samakovlis et al., 1990).

In order to compare regulation of GFP expression with that of the resident metchnikowin gene, we performed quantitative measurements. First we compared the kinetics of RNA synthesis induction on the resident metchnikowin gene and the GFP reporter gene by Northern blot analysis. RNA was extracted from transgenic flies before or at various time points after pricking with a bacteria-soaked needle, and analyzed by Northern blotting using metchnikowin and GFP probes. As shown in Figure 6(a), GFP was induced upon immune challenge with kinetics similar to metchni- kowin: RNA transcripts could be detected three hours after stimulation, and reached a plateau after 14 hours. Expression levels started to slowly decrease after 24 hours. We also examined GFP expression at the protein level by Western blotting, and found that the protein started to be detectable six hours after stimulation, and accumulated until 72 hours after stimulation, before levelling off (Figure 6(b)). This accumulation reflects the high stability of the GFP (Cubitt et al., 1995). We next measured the intensity of fluorescence in immunechallenged flies. Fluorescence could be detected as early as six hours after induction, and reached a maximum at 72 hours, in good agreement with the Western blot data (Figure 7(a)). These experiments were performed on a representative transgenic strain. Quantification of fluorescence intensity for four other independent lines is presented in Figure 7(a), and shows only moderate variability from line to line, corresponding to positional effects. These data indicate that $1.5 \mathrm{~kb}$ of metchnikowin gene $5^{\prime}$ flanking sequences contain all the elements necessary to confer full inducibility on a reporter gene upon bacterial challenge in transgenic flies.

As discussed above, metchnikowin is expressed in the absence of immune stimulus in Toll gain-of-

Figure 4. Organization of the metchnikowin gene. (a) Schematic representation and restriction map of a $2.5 \mathrm{~kb}$ SalIXhoI genomic fragment comprising the metchnikowin cDNA. The coding region is marked by a hatched box (ORF), the arrow indicates the $5^{\prime}$ to $3^{\prime}$ orientation of the transcribed sequence. The size of the genomic fragment in base-pairs (bp) is shown below. (b) Sequence of the metchnikowin gene. The extent of the longest cDNA (Levashina et al., 1995) is shown (cDNA) together with the amino acid sequence (one letter code). The mature peptide sequence is marked in bold type. Asterisks indicate the putative sites of cleavage of a signal peptide. Broken lines correspond to the putative polyadenylation sites. The start site (arrow) and the putative TATA box (boxed) are marked as well as the three putative Rel sites (underlined) located on either strand. (c) Schematic organization of the metchnikowin promoter. The open reading frame is represented by a hatched box, the arrow indicates the transcriptional start point. The location of sequence motifs identical to Interferon Consensus Response Elements (ICRE), Rel sites (Rel), NF-IL6 response elements (NF-IL6 RE), or GATA sites (GATA) are shown. 
(a)

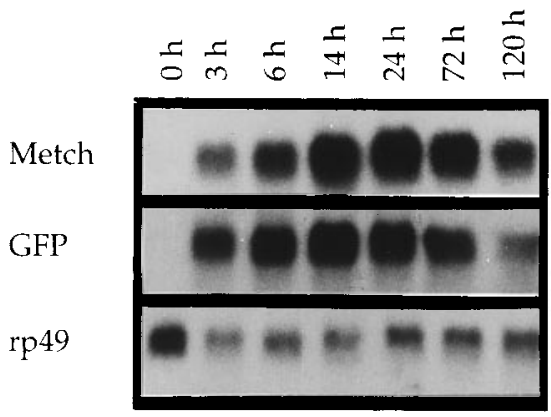

(b)

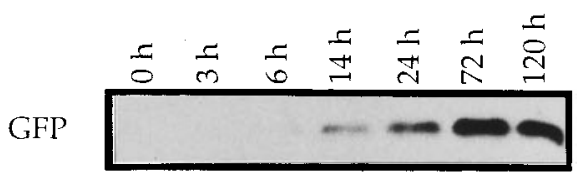

Figure 6. Inducibility of GFP expression in transgenic Drosophila upon immune challenge. (a) Northern blot analysis: total RNA was extracted from transgenic Drosophila either untreated ( 0 hour) or at various time points post-immunization with a mixture of Gram-positive and Gram-negative bacteria, and analyzed by Northern blot. The blot was first probed with the metchnikowin cDNA, and after dehybridization successively with the GFP and rp49 cDNAs. (b) Western blot analysis: protein extracts were prepared from transgenic Drosophila (line 1) untreated (0 hour) or at various times post-immune challenge and analyzed by Western blotting using an anti-GFP antibody.

function mutants. In order to check whether the $1.5 \mathrm{~kb}$ of upstream sequences from the metchnikowin gene fused to the GFP cDNA are sufficient to mimick regulation of the endogenous metchnikowin gene by the TOLL pathway, we crossed two independent transgenic lines containing the metchnikowin-GFP reporter construct with a strain carrying a gain-of-function $T l$ allele. As shown in Figure 5(c), the resulting larvae exhibited fluorescence in the fat body in the absence of immune challenge. The fact that our reporter construct contains $3^{\prime}$ flanking sequences from the drosomycin gene raises the possibility that TOLL might induce GFP expression through these sequences. To rule this out, similar experiments were performed with transgenic flies in which the diptericin or drosocin promoter was placed upstream of the GFP reporter cassette containing the same drosomycin terminator sequences. When these reporter genes were put in $T l^{D}$ context, no fluorescence could be detected in the larval fat body (S. Ohresser et al., unpublished results), thus implying that the target sequences for TOLL signaling are in the metchnikowin upstream regulatory sequences and not in the drosomycin terminator.

Recent studies in our laboratory have shown that natural infection of adult Drosophila with the entomopathogenic fungus Beauveria bassiana results in the selective induction of the genes encoding the peptides with antifungal activity: drosomycin and metchnikowin (Lemaitre et al., 1997). Transgenic flies containing the metchnikowin promoter-GFP fusion gene, as well as transgenic flies containing a drosomycin promoter-GFP (Ferrandon et al., 1998) or a drosocin-GFP (S. Ohresser et al., unpublished results) fusion gene, were covered with $B$. bassiana spores, and placed at $29^{\circ} \mathrm{C}$. Fluorescence intensity was measured 72 hours later. A strong fluorescence could be detected in challenged transgenic flies containing the drosomycin or metchnikowin promoter fused to the GFP coding sequences. In contrast, no induction of fluorescence following fungal infection could be observed in transgenic flies expressing GFP under the control of the drosocin promoter (Figure 7(b)).

Altogether, these data strongly suggest that $1.5 \mathrm{~kb}$ of upstream sequences from the metchnikowin gene are sufficient to confer proper inducibility on a reporter gene in transgenic Drosophila upon immune challenge, or in $\mathrm{Tl}^{\mathrm{D}}$ context. Further analysis of these sequences in vivo and in vitro should help us to understand how the immune response is triggered by the TOLL pathway and the imd gene product.

\section{Discussion}

\section{Metchnikowin gene expression can be regulated by either the imd gene product or the TOLL pathway}

Here we describe the regulation of metchnikowin gene expression in wild-type and mutant Drosophila, and provide evidence that this gene can be regulated independently by the TOLL or the IMD pathway. Analysis of the expression pattern of antimicrobial peptide genes in mutants defective either for the TOLL pathway or the imd gene enables us to divide them into four groups. The first category of genes is exemplified by the gene for drosomycin, which appears to be regulated mainly by the TOLL pathway. The second category of genes, to which the genes encoding diptericin and drosocin belong, appears to be regulated mainly by the imd gene product. The third category of genes encompasses the cecropin A, attacin and defensin genes, which require both the TOLL pathway and IMD for full inducibility upon immune challenge (Lemaitre et al., 1996). The fourth category, which we describe here, is represented to date only by the gene encoding metchnikowin. Inducibility of this gene by immune challenge is not affected in spz-, Tl-, tub- or pll-deficient mutants, and is only slightly reduced in imd mutants. However, this gene is no longer immuneinducible in strains in which both pathways are inactivated. Thus, the TOLL pathway is to some extent sufficient for the induction of metchnikowin expression in imd mutants, while the IMD pathway appears sufficient to achieve this goal in $\mathrm{Tl}$ deficient mutants. In keeping with this interpretation, we observed that the mutations causing constitutive activation of the TOLL pathway $\left(\mathrm{Tl}^{\mathrm{D}}\right.$ and $\mathrm{cact}^{-}$) reproducibly resulted in low but significant 
(a)

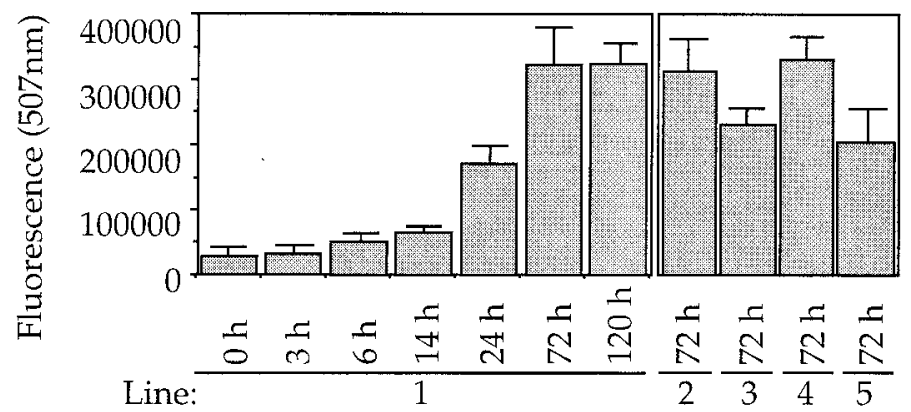

(b)

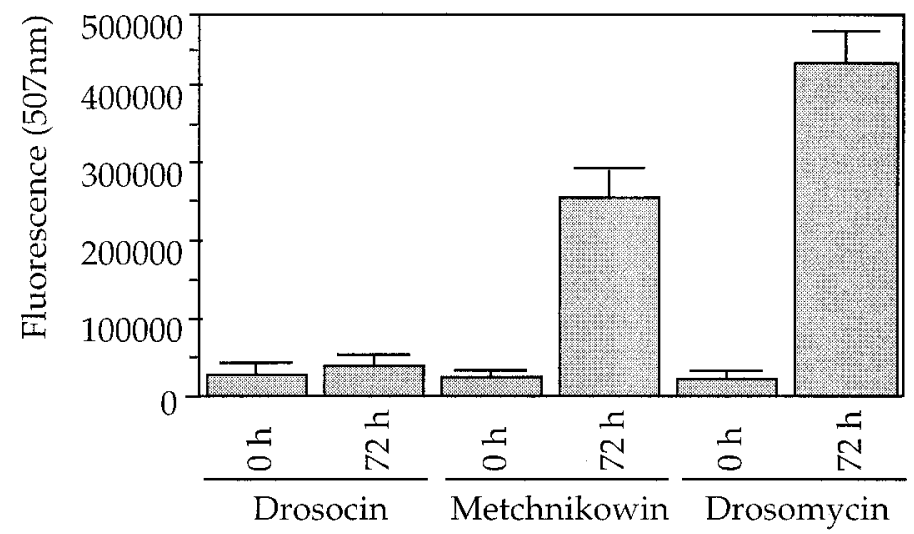

Figure 7. Inducibility of GFP fluorescence in transgenic Drosophila upon immune challenge. (a) Intensity of GFP fluorescence in protein extracts prepared from transgenic Drosophila untreated (0 hour) or at various time points after challenge with bacteria. The level of fluorescence 72 hours after immune challenge is compared for five independent transgenic lines. (b) Induction of GFP expression by natural infection with $B$. bassiana. Transgenic flies expressing GFP under the control of the drosocin, metchnikowin or drosomycin promoters (four groups of ten flies for each reporter construct) were left untreated (0 hour) or covered with spores of $B$. bassiana. Protein extracts were prepared 72 hours later, and fluorescence emission at $507 \mathrm{~nm}$ was measured. Data represent means \pm standard deviations. expression of metchnikowin in the absence of any immune challenge (see Figures 1 and 3). As for the genes of the third category, both the TOLL and the IMD pathways participate in metchnikowin regulation, but in this case the two pathways regulate gene expression independently of one another.

The existence of two distinct regulatory pathways controlling the expression of antimicrobial genes points to the existence of distinct recognition mechanisms. Recent data indicate that the Drosophila immune system can discriminate among various classes of microorganisms (Lemaitre et al., 1997). When different categories of microorganisms were injected into flies, Gram-negative bacteria were found to preferentially induce antibacterial genes, while drosomycin was strongly induced by fungi and to a lesser extent by Gram-positive bacteria. Most interestingly, metchnikowin gene expression was found to be strongly inducible by both bacteria and fungi (Lemaitre et al., 1997). In addition, when flies are naturally infected with the entomopathogenic fungus $B$. bassiana, in the absence of pricking and the non-specific effects of injury, only drosomycin and, to a lesser extent, metchnikowin are induced, while the genes encoding antibacterial peptides remain silent (Lemaitre et al., 1997; and Figure 7(b)). It is therefore now established that Drosophila can discriminate between microorganisms of fungal or bacterial origin, and, at least in the case of fungal pathogens, mount an appropriate response by selectively turning on the genes encoding peptides with antifungal activity (e.g. drosomycin, metchnikowin). These data suggest that the two pathways that participate in the regulation of metchnikowin expression can be turned on independently, by the recognition of specific microorganisms, and agree well with what we know from the function of metchnikowin, since this peptide is active against both bacteria and fungi.

Altogether, these experiments indicate that the complex interplay between two pathways allows for the selective induction of subsets of genes during the immune response in Drosophila, and suggest that this specificity results from differences in the organization of the promoters of the genes encoding the antimicrobial peptides.

\section{Organization of the metchnikowin promoter}

We have cloned and sequenced $1.5 \mathrm{~kb}$ of upstream sequences from the metchnikowin gene. In order to determine if these sequences are necessary and sufficient for metchnikowin gene expression, transgenic strains were constructed, in which the reporter gene encoding GFP was placed under the control of this DNA fragment. Analysis of the resulting flies and larvae indicates that we have cloned the metchnikowin promoter: first, the $1.5 \mathrm{~kb}$ fragment confers inducibility to the GFP gene upon immune challenge; second, the kinetics of inducibility of GFP expression parallel those of metchnikowin mRNA expression with a peak of expression at 14 hours; third, in larvae carrying the gain-of-function mutation $\mathrm{Tl}^{10 b}$, which constitu- 
tively activates the TOLL pathway, the GFP is expressed in the fat body in the absence of immune challenge; fourth, natural infection by $B$. bassiana induces GFP expression in the fat body of transgenic flies. We therefore conclude that this DNA fragment contains the regulatory elements necessary for the control of metchnikowin expression in response to immune challenge or stimulation by the TOLL pathway.

Sequence analysis of the metchnikowin promoter revealed several features in common with the promoters of insect genes coding for other antimicrobial peptides, and of mammalian genes expressed during the acute phase response (Hoffmann \& Reichhart, 1997). The most striking feature is the presence of sequence motifs recognized by Rel family members (Rel sites). These sites are present in most mammalian genes involved in inflammatory responses, and bind the inducible transactivator NF-kB (Siebenlist et al., 1994). These sites have also been described in all the promoters of antimicrobial peptide genes characterized to date in Drosophila. Three Rel sites are present in the metchnikowin promoter at positions -63, -176 and -638 . Interestingly, one of these sites is located relatively far upstream from the cap site, whereas for most antimicrobial peptide genes Rel sites are located in the near upstream region. It is worth noting here that, in the case of the drosomycin promoter, the Rel sites that are likely to be functionally important are located between -500 and -800 (L. Michaut et al., unpublished results). To date, the strongest evidence that Rel proteins are involved in the control of antimicrobial gene expression in Drosophila comes from the results obtained with cact-deficient mutants. Indeed, CACTUS/I $\kappa \mathrm{B}$ proteins retain Rel family members in the cytoplasm (Siebenlist et al., 1994); hence the expression of drosomycin (Lemaitre et al., 1996) and metchnikowin (this paper) in the absence of immune challenge in cactus-deficient mutants suggests that a Rel protein (possibly the DORSAL-related factor DIF (Ip et al., 1993), or DIF in association with DORSAL, both of which are expressed in the fat body) translocates to the nucleus to activate the genes encoding these two peptides. The fact that the antibacterial peptides (e.g. diptericin, drosocin, attacin, cecropin A1 or defensin) are not expressed in such cact-deficient mutants (Lemaitre et al., 1996) suggests a role for a Rel protein that does not interact with CACTUS, such as RELISH (Dushay et al., 1996). Alternatively, DORSAL or DIF might need an additional challenge-dependent modification to become active on these promoters.

In addition to Rel sites, the metchnikowin promoter contains sequence motifs homologous to cisacting regulatory elements binding the mammalian transcription factors GATA (Orkin, 1992), NF-IL6 (Hocke et al., 1992), as well as several GGAAANN sequence motifs present in the interferon consensus response elements of mammalian interferoninduced genes (Georgel et al., 1995). In mammals, cooperative interaction of $\mathrm{NF}-\kappa \mathrm{B}$ with multiple transcription factors is well documented (Siebenlist et al., 1994). The few studies that have dissected the organization of immune-inducible promoters in Drosophila reveal similar features. Footprinting and gel-shift experiments performed on the diptericin promoter have shown that the proximal upstream region of the gene contains multiple functional regulatory sequence elements (Georgel et al., 1993; Kappler et al., 1993). The functional importance of these elements was demonstrated by analysis of transgenic strains of Drosophila bearing mutations in these sequence motifs (Meister et al., 1994). Interestingly, the most proximal of the regulatory region was shown to contain two motifs overlapping with the Rel sites, and similar in sequence to NF-IL6 response elements and a core motif recognized by interferon regulatory factor 1 (IRF-1). The cecropin A1 gene promoter, which has also been studied in detail, similarly contains conserved sequence motifs adjacent to the Rel site (Engstrom et al., 1993; Tryselius et al., 1992). Gel shift experiments and analysis of mutations in transient transfection assays have recently shown that one of these motifs, AGATAA, is functionally important; this motif is identical to the consensus sequence WGATAR, recognized in mammalian promoters by transcriptional activators of the GATA family (Kadalayil et al., 1997). In the metchnikowin promoter, the most distal Rel site overlaps with a GGAAANN motif, and is adjacent to an NF-IL6 response element, a situation reminiscent of the organization of the proximal element in the diptericin promoter (Georgel et al., 1993; Meister et al., 1994). Another Rel site, at position -176 , is located $11 \mathrm{bp}$ downstream of a GATA site, a situation reminiscent of the cecropin promoter (Kadalayil et al., 1997). A GATA site is also present $8 \mathrm{bp}$ upstream of a Rel site in the drosocin gene promoter (Charlet et al., 1996). Finally, the third, most proximal, Rel site is not in the vicinity of any of the response elements discussed above. Functional characterization of these sequence motifs, and purification of the proteins that recognize them, should enable us to understand how the antimicrobial genes promoters are regulated.

The picture that is emerging from the study of the organization of the promoters of immuneinducible peptides in Drosophila therefore suggests a situation in which Rel proteins combine with other DNA binding factors, the nature of which determines the selection of specific Rel sites. Activation of different subsets of Rel proteins and different accessory factors by specific microorganisms might explain the selective induction of some genes during the immune response in Drosophila. It will be of interest to determine whether different Rel sites are responsible for induction of metchnikowin expression by the TOLL and IMD pathways, or if both pathways converge on a single Rel site, and activate different accessory factors. 


\section{Materials and Methods}

\section{Drosophila stocks}

The lines used in this study have been described (Lemaitre et al., 1995, 1996). In order to demonstrate the inducibility of the metchnikowin promoter by the TOLL pathway, transgenic females containing the GFP gene under the control of the metchnikowin promoter were crossed with males heterozygous for the $T l^{10 b}$ allele. Crosses were performed at $25^{\circ} \mathrm{C}$, and third instar larvae were collected. $\mathrm{w}^{-}$flies were used as wild-type flies, and were also used as recipients for transformation.

\section{Immunization procedures}

Bacterial challenge was performed by pricking adults (3 to 5-day-old) with a thin needle previously dipped into a concentrated bacterial culture of Escherichia coli and Micrococcus luteus. Flies were then kept at $25^{\circ} \mathrm{C}$, and were snap frozen in liquid nitrogen after different time intervals. For fungal infection, flies were anesthetized and covered with spores of B. bassiana (strain 80.2). They were then placed at $29^{\circ} \mathrm{C}$, and collected for fluorescence quantification 48 hours later.

\section{RNA analysis}

Total RNA extraction and Northern blotting experiments were performed as described (Lemaitre et al., 1995). The following probes were used: cecropin A1 cDNA (Kylsten et al., 1990); diptericin cDNA (Wicker et al., 1990); drosomycin cDNA (Fehlbaum et al., 1994); metchnikowin cDNA (Levashina et al., 1995); and rp49 cDNA (O'Connell \& Rosbach, 1984). Hybridization signals were quantified using a Bio-Imager system, and the signals obtained for immune genes RNA were normalized with the corresponding value of the ubiquitously expressed ribosomal protein rp49 RNA signal.

\section{Isolation and sequencing of the metchnikowin gene}

A total of 100,000 plaque-forming units of an amplified Drosophila Oregon R genomic library constructed in $\lambda$ DASH II vector (gift from Dr J.-A. Lepesant) were screened with a ${ }^{32} \mathrm{P}$-labeled $280 \mathrm{bp}$ cDNA probe (insert A1; Levashina et al., 1995). Hybridization of nitrocellulose filters and washing were performed as described (Dimarcq et al., 1994). Nine hybridization-positive plaques were obtained and shown by restriction mapping to correspond to four overlapping genomic fragments. A $9 \mathrm{~kb}$ SalI fragment was cloned in the pTZ18R phagemid by standard methods (Sambrook et al., 1989). After restriction mapping a $2.5 \mathrm{~kb}$ SalI-XhoI fragment was subcloned into a M13mp18 vector. Sequencing was performed by the dideoxy method of Sanger et al. (1977) using a Pharmacia Sequencing kit. The products were separated on a polyacrylamide gel $(6 \%$ acrylamide, $8 \mathrm{M}$ urea). Routine computer analysis of the sequencing data was facilitated by DNASTAR ${ }^{\mathrm{TM}}$ software for a Macintosh microcomputer. The putative cis-regulatory motifs were determined using the MatInspector V2.1 program (Copyright"1997Transfac-Team).

\section{Construction of the metchnikowin reporter gene and isolation of transformed fly lines}

The F64L mutation (replacement of the phenylalanine residue in position 65 by a leucine) in GFP was introduced by PCR-directed mutagenesis (Higuchi et al., 1988) using the high fidelity Vent polymerase (New England Biolabs). The plasmid pJM705 (Ferrandon et al., 1998), which contains the S65 T version of GFP, served as a template. The PCR fragment was sequenced to control the fidelity of the reaction. The modified GFP cDNA was fused to a $790 \mathrm{bp}$ fragment of drosomycin gene $3^{\prime}$ flanking region (Ferrandon et al., 1998). A fragment containing metchnikowin gene sequences from position -1497 (SalI restriction site) to position +32 was subsequently fused upstream of the GFP sequences. The complete metchnikowin $5^{\prime}$ sequences-GFP-drosomycin $3^{\prime}$ flanking region cassette was then subcloned into the transformation vector pCasper, before injection into embryos. The helper plasmid p $\pi \mathrm{q} 2-3$ was co-injected with the pCasper vector containing the fusion gene into $\mathrm{w}^{-}$embryos, and transformants were recovered as G1 $\mathrm{w}^{+}$flies. Crosses with flies carrying appropriate balancers were performed to establish stable heterozygous or homozygous lines, as well as to determine the chromosome carrying the insertion. Nine insertions were found on the second chromosome, and ten on the third chromosome. The established lines were maintained at $25^{\circ} \mathrm{C}$ on a standard cornmeal medium.

\section{Quantification of GFP expression}

For Western blot analysis, $20 \mu \mathrm{g}$ of protein extract prepared from transgenic female flies at various time points after immunization were resuspended in Laemmli buffer, and analyzed by SDS-PAGE on a $10 \%$ acrylamide gel (Laemmli, 1970). After migration, the proteins were electroblotted onto a nitrocellulose filter, which was probed (overnight, $4^{\circ} \mathrm{C}$ ) with a polyclonal rabbit anti-GFP antibody (Clontech), at a 1:2000 dilution in Tris-buffered saline (TBS) supplemented with $0.3 \%$ low fat dry milk and $0.05 \%$ Tween 20 . A horseradish peroxidase-antirabbit conjugate (Amersham Life Science) was used as a second antibody at a dilution of 1:10,000, and was incubated with the filter for one hour at $37^{\circ} \mathrm{C}$. The GFP was detected using the enhanced chemiluminescence technique (Amersham Life Science).

Fluorescent GFP in live flies was visualized using a photoautomat (wild MPS48/52, Leica) equiped for fluorescence microscopy. For quantitative measurements of GFP activity, ten female adults were homogenized in Chalfie buffer (Chalfie et al., 1994), and extracts were centrifuged for ten minutes at $10,000 \mathrm{~g}$ to remove debris. Fluorescence in the supernatant was measured with a spectrofluorimeter (Jobin Yvon fluoromax-2). The emission spectra were measured from $495 \mathrm{~mm}$ to $560 \mathrm{~nm}$, with a fixed excitation wavelength of $495 \mathrm{~nm}$. The peak of emission was observed at $507 \mathrm{~nm}$.

\section{Acknowledgments}

E. A. Levashina and S. Ohresser made equal contributions to this work. We thank Dominique Ferrandon for injection into embryos as well as for his many advice; Jean-Marc Reichhart for stimulating discussions; Jules A. Hoffmann for continued support and interest, 
as well as critical reading of the manuscript. The technical expertise of Reine Klock and Marie Eve Moritz is gratefully acknowledged. This work was supported in part by a grant from the Human Frontiers Science Program.

The nucleotide sequence for the metchnikowin gene has been submitted to GenBank and will be accessible under the accession number AF030959.

\section{References}

Asling, B., Dushay, M. \& Hultmark, D. (1995). Identification of early genes in the Drosophila immune response by PCR-based differencial display: the Attacin A gene and the evolution of attacin-like proteins. Insect Biochem. Mol. Biol. 25, 511-518.

Bulet, P., Dimarcq, J., Hetru, C., Lagueux, M., Charlet, M., Hegy, G., Van Dorsselaer, A. \& Hoffmann, J. (1993). A novel inducible antibacterial peptide of Drosophila carries an O-glycosylated substitution. J. Biol. Chem. 268, 14893-14897.

Cao, Z., Henzel, W. \& Gao, X. (1996). IRAK: a kinase associated with the interleukin-1 receptor. Science, 271, 1128-1131.

Chalfie, M., Tu, Y., Euskirchen, G., Ward, W. \& Prasher, D. (1994). Green fluorescent protein as a marker for gene expression. Science, 263, 802-805.

Charlet, M., Lagueux, M., Reichhart, J., Hoffmann, D., Braun, A. \& Meister, M. (1996). Cloning of the gene encoding the antibacterial peptide drosocin involved in Drosophila immunity. Expression studies during the immune response. Eur. J. Biochem. 241, 699-706.

Cubitt, A., Heim, R., Adams, S., Boyd, A., Gross, L. \& Tsien, R. (1995). Understanding, improving and using green fluorescent proteins. Trends Biochem. Sci. 20, 448-455.

Dimarcq, J., Hoffmann, D., Meister, M., Bulet, P., Lanot, R., Reichhart, J. \& Hoffmann, J. (1994). Characterization and transcriptional profiles of a Drosophila gene encoding an insect defensin. A study in insect immunity. Eur. J. Biochem. 221, 201-209.

Dushay, M., Asling, B. \& Hultmark, D. (1996). Origins of immunity: Relish, a compound Rel-like gene in the antibacterial defense of Drosophila. Proc. Natl Acad. Sci. USA, 93, 10343-10347.

Engstrom, Y., Kadayalil, L., Sun, S., Samakovlis, C., Hultmark, D. \& Faye, I. (1993). kB-like motifs regulate the induction of immune genes in Drosophila. J. Mol. Biol. 232, 327-333.

Fehlbaum, P., Bulet, P., Michaut, L., Lagueux, M., Broeckaert, W., Hetru, C. \& Hoffmann, J. (1994). Insect immunity: septic injury of Drosophila induces the synthesis of a potent antifungal peptide with sequence homology to plant antifungal peptides. J. Biol. Chem. 269, 33159-33163.

Ferrandon, D., Jung, A., Criqui, M., Lemaitre, B., Uttenweiler-Joseph, S., Michaut, L., Reichhart, J. \& Hoffmann, J. (1998). A drosomycin-GFP reporter transgene reveals a local immune response in Drosophila that is not dependent on the Toll pathway. EMBO J, 17, 1217-1227.

Gay, N. \& Keith, F. (1991). Drosophila Toll and IL-1 receptor. Nature, 351, 355-356.

Georgel, P., Meister, M., Kappler, C., Lemaitre, B., Reichhart, J. \& Hoffmann, J. (1993). Insect immu- nity: the diptericin promoter contains multiple functional regulatory sequences homologous to mammalian acute-phase response elements. Biochem. Biophys. Res. Commun. 197, 508-517.

Georgel, P., Kappler, C., Langley, E., Gross, I., Nicolas, E., Reichhart, J. \& Hoffmann, J. (1995). Drosophila immunity. A sequence homologous to mammalian interferon consensus response element enhances the activity of the diptericin promoter. Nucl. Acids Res. 23, 1140-1145.

Higuchi, R., Krummel, B. \& Saiki, R. K. (1988). A general method of in vitro preparation and specific mutagenesis of DNA fragments: study of protein and DNA interactions. Nucl. Acids Res. 16, 73517364 .

Hocke, G., Barry, D. \& Fey, G. (1992). Synergistic action of interleukin- 6 and glucocorticoids is mediated by the interleukin- 6 response element of the rat alpha 2 macroglobulin gene. Mol. Cell Biol. 12, 2282-2294.

Hoffmann, J. \& Reichhart, J. (1997). Drosophila immunity. Trends Cell Biol. 7, 309-316.

Ip, Y., Reach, M., Enstrom, Y., Kadalayil, L., Cai, H., Gonzalez-Crespo, S., Tatei, K. \& Levine, M. (1993). Dif, a dorsal-related gene that mediates an immune response in Drosophila. Cell, 75, 753-763.

Kadalayil, L., Petersen, U. \& Engstrom, Y. (1997). Adjacent GATA and kB-like motifs regulate the expression of a Drosophila immune gene. Nucl. Acids Res. 25, 1233-1239.

Kappler, C., Meister, M., Lagueux, M., Gateff, E., Hoffmann, J. \& Reichhart, J. (1993). Insect immunity. Two $17 \mathrm{bp}$ repeats nesting a $\kappa$ B-related sequence confer inducibility to the diptericin gene and bind a polypeptide in bacteria-challenged Drosophila. EMBO J. 12, 1561-1568.

Kylsten, P., Samakovlis, C. \& Hultmark, D. (1990). The cecropin locus in Drosophila: a compact gene cluster involved in the response to infection. EMBO J. 9, 217-224.

Laemmli, U. K. (1970). Cleavage of structural proteins during the assembly of the head of bacteriophage T4. Nature, 227, 680-685.

Lemaitre, B., Kromer-Metzger, E., Michaut, L., Nicolas, E., Meister, M., Georgel, P., Reichhart, J. \& Hoffmann, J. (1995). A recessive mutation, immune deficiency (imd), defines two distinct control pathways in the Drosophila host defense. Proc. Natl Acad. Sci. USA, 92, 9365-9469.

Lemaitre, B., Nicolas, E., Michaut, L., Reichhart, J. \& Hoffmann, J. (1996). The dorsoventral regulatory gene cassette spätzle/Toll/cactus controls the potent antifungal response in Drosophila adults. Cell, 86, 973-983.

Lemaitre, B., Reichhart, J. \& Hoffmann, J. (1997). Drosophila host defense: differential induction of antimicrobial peptide genes after infection by various classes of microorganisms. Proc. Natl Acad. Sci. USA, 94, 14614-14619.

Levashina, E., Ohresser, S., Bulet, P., Reichhart, J., Hetru, C. \& Hoffmann, J. (1995). Metchnikowin, a novel immune-inducible proline-rich peptide from Drosophila with antibacterial and antifungal properties. Eur. J. Biochem. 233, 694-700.

Meister, M., Braun, A., Kappler, C., Reichhart, J. M. \& Hoffmann, J. A. (1994). Insect immunity. A transgenic analysis in Drosophila defines several functional domains in the diptericin promoter. $E M B O \mathrm{~J}$. 13, 5958-5966. 
Morisato, D. \& Anderson, K. (1995). Signaling pathways that establish the dorsal-ventral pattern of the drosophila embryo. Annu. Rev. Genet. 29, 371-399.

O'Connell, P. \& Rosbach, M. (1984). Sequence, structure and codon preference of the Drosophila ribosomal protein 49 gene. Nucl. Acids Res. 12, 5495-5513.

Orkin, S. (1992). GATA-binding transcription factors in hematopoietic cells. Blood, 80, 575-581.

Samakovlis, C., Kimbrell, D., Kylsten, P., Engstrom, A. \& Hultmark, D. (1990). The immune response in Drosophila: pattern of cecropin expression and biological activity. EMBO J. 9, 2969-2976.

Sambrook, J., Fritsch, E. \& Maniatis, T. (1989). Molecular Cloning: A Laboratory Manual, 2nd edit., Cold Spring Harbor Laboratory Press, Plain View, NY.
Sanger, F., Nicklen, S. \& Coulson, A. R. (1977). DNA sequencing with chain-terminating inhibitors. Proc. Natl Acad. Sci. USA, 74, 5463-5467.

Siebenlist, U., Franzoso, G. \& Brown, K. (1994). Structure, regulation and function of NF-кB. Апnи. Rev. Cell Biol. 10, 405-455.

Tryselius, Y., Samakovlis, C., Kimbrell, D. \& Hultmark, D. (1992). CecC, a cecropin gene expressed during metamorphosis in Drosophila pupae. Eur. J. Biochem. 204, 395-399.

Wicker, C., Reichhart, J., Hoffmann, D., Hultmark, D., Samakovlis, C. \& Hoffmann, J. (1990). Insect immunity: characterization of a Drosophila cDNA encoding a new member of the diptericin promoter. J. Biol. Chem. 265, 22493-22498.

Edited by M. Yaniv

(Received 28 October 1997; received in revised form 5 February 1998; accepted 6 February 1998) 